\title{
Two-stage Artificial Intelligence Clinical Decision Support System for Cardiovascular Assessment using Convolutional Neural Networks and Decision Trees
}

\author{
Shahab Pasha ${ }^{1} \mathbb{C}^{\mathrm{a}}$, Jan Lundgren ${ }^{1} \mathbb{C}^{\mathrm{b}}$ Marco Carratù $^{2}$, Patrik Wreeby ${ }^{3}$ and Consolatina Liguori ${ }^{2}$ \\ ${ }^{I}$ STC Research Centre, Mid Sweden University, Sundsvall, Sweden \\ ${ }^{2}$ Department of Industrial Engineering, University of Salerno, Fisciano, Italy \\ ${ }^{3}$ Premicare AB, Sörberge, Sweden
}

Keywords: Artificial Intelligence, Cardiovascular Assessment, Decision Trees, Deep Learning, Feature Selection.

\begin{abstract}
This paper describes an artificial-intelligence-assisted screening system implemented to support medical cardiovascular examinations performed by doctors. The proposed system is a two-stage supervised classifier comprising a convolutional neural network for heart murmur detection and a decision tree for classifying vital signs. The classifiers are trained to prioritize higher-risk individuals for more time-efficient assessment. A feature selection approach is applied to maximize classification accuracy by using only the most significant vital signs correlated with heart issues. The results suggest that the trained convolutional neural network can learn and detect heart sound anomalies from the time-domain and frequency-domain signals without using any user-guided mathematical or statistical features. It is also concluded that the proposed two-stage approach improves diagnostic reliability and efficiency.
\end{abstract}

\section{INTRODUCTION}

The healthcare sector is entering the age of global artificial intelligence (AI) convergence (Morsy, 2018). Benefiting from mobile applications and largescale data, technologies such as Blue Button (website of the Office of the National Coordinator for Health Information Technology, n.d.) and IBM Watson (IBM Watson, n.d.) help track treatment progress and reduce wrong diagnoses. In healthcare, AI covers a wide range of applications, including screening (Lin, Chang, Lin, Tsai, \& Chen, 2017), monitoring (Ivascu, Cincar, \& Negru, 2017), and diagnosis (Islam Chowdhuryy, Sultana, Ghosh, Ahamed, \& Mahmood, 2018). Of the applied AI methods, deep learning techniques (Gharehbaghi \& Lindén, 2017; Loh \& Then, 2017) seem to be more adaptable, accurate, and robust in a wide range of applications and biological signals, such as lung sound classification (Chen, Zhang, Tian, Zhang, Chen, \& Lei, 2016), cardiac auscultation (Amiriparian, Schmitt, M., Cummins, N., Qian, K., Dong, F., \& Schuller, 2018), phonocardiography (Thomae \&

a(D) https://orcid.org/0000-0002-6805-166X

b(iD) https://orcid.org/0000-0003-1819-6200
Dominik, 2016), and vital sign evaluation (Jones, 2013).

Biological signals (e.g., heart sound signals) with cyclic characteristics often display nonstationary behavior not only within but across cycles in cycleto-cycle variation. This gives the signal a high level of complexity, making classifier development a major challenge.

Unlike in many industrial applications, the origin of the complexities in most biological signals has yet to be fully understood (Gharehbaghi \& Lindén, 2017).

AI-assisted methods applied to biological signals can be categorized into four main categories: neural network-based and deep learning classification (Zabihi, Rad, Kiranyaz, Gabbouj, \& Katsaggelos, 2016), support vector machine-based classification (Barhatte, Ghongade, \& Thakare, 2015), hidden Markov-model-based classification (Vernekar, Nair, Vijaysenan, \& Ranjan, 2016), and clustering-based classification (Clifford, 2016). Advanced deep learning techniques can automatically extract salient patterns directly from the input (i.e., training set) and use the produced knowledge to classify unseen samples (Shao, $\mathrm{Wu}, \& \mathrm{Li}, 2014)$. This capability of 


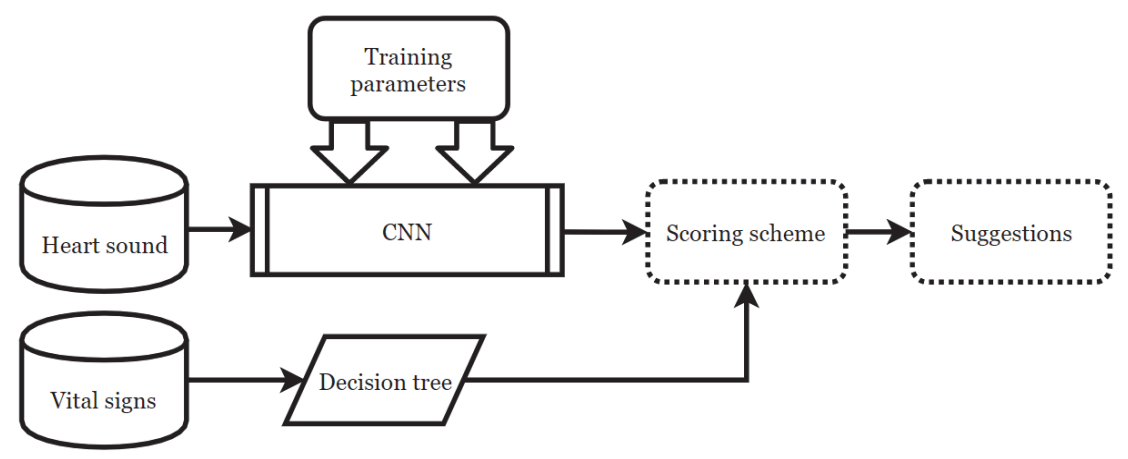

Figure 1: The two stage AI decision support system.

deep networks makes them the right tool with which to extract and learn particular patterns from diverse and relatively large training sets, such as heart sound databases (PhysioNet, 2016). However most existing AI-assisted heart sound analysis systems use medically incomprehensible mathematical and statistical features, such as wavelets (Clifford, 2016), Stockwell transformation (Moukadem, Dieterlen, \& Brandt, 2013), Mel-frequency cepstral coefficients (MFCCs) (Chen, 2017), and the likelihood function (Yamashita, Himeshima, \& Matsunaga, 2014).

In this research, a convolutional neural network $(\mathrm{CNN})$ is trained based on the time-domain and highresolution frequency-domain "normal" and "abnormal" heart sound signals (PhysioNet, 2016). Along with the heart sound signals, the vital signs and patient information are analyzed by a C4.5 decision tree as side information for a comprehensive cardiovascular assessment. An optimization process (Visalakshi \& Radha, 2014) is applied to find the optimal subset of vital signs yielding the highest classification accuracy. The proposed system is designed for making suggestions to support the clinical assessment process. The system uses signal processing and data-mining techniques to maximize accuracy, using filters designed to find the most significant frequency band for heart murmurs and to find the optimized set of vital signs.

The remainder of this paper is organized as follows. Section 2 briefly describes the cardiovascular examination procedure as performed by medical experts. Section 3 describes the proposed AI system and the deep network structure replicating the medical procedure described in section 2. In section 4 , the proposed two-stage system is trained and evaluated. The paper concludes in section 5 .

\section{CARDIVASCULAR EXAMINATION AND THE HEART SOUND MODEL}

During a cardiovascular examination, the doctor has the patient assume either a lying supine or a sitting position and starts with the general examination, which includes measuring body temperature, blood pressure, skin hydration, pulse, and blood oxygen saturation. Heart sound auscultation with a stethoscope is the next step after the general examination. The doctor listens to the heart at the apex, base (i.e., the part of the heart between the apex and sternum), and in the aortic and pulmonary areas.

A normal heart sound consists of two fundamental sounds referred to as $S 1$ and $S 2 . S 1$ occurs when the mitral and tricuspid valves close and $S 2$ occurs when the aortic and pulmonic valves close. Other sounds include the third heart sound $(S 3)$, the fourth heart sound (S4), the systolic ejection click (EC), the midsystolic click (MC), the diastolic sound or opening snap (OS), and heart murmurs. Heart murmurs (Vepa, 2009) made by turbulent blood flow in the heart and blood leakage through the closed valves are a stationary low-frequency noise that indicates underlying heart issues. This might happen during the delay between $S 1$ and $S 2$ (i.e., the systole) or between two consecutive beats (i.e., the diastole).

The heart sound signal is a low-frequency signal covering a range of frequencies from 20 to $500 \mathrm{~Hz}$, so a relatively low sampling frequency, i.e. $2000 \mathrm{~Hz}$ according to the PhysioNet database (PhysioNet, 2016), is sufficient.

The generated heart sound traveling through the chest and being distorted by the respiratory noise is mathematically modeled as:

$$
\begin{gathered}
x(n)=h^{s}(n) * s(n)+h^{l}(n) * l(n) \\
+v(n),
\end{gathered}
$$


where $x(n)$ is the signal detected by the stethoscope, $s(n)$ the heart sound, $l(n)$ the bronchial/vesicular lung sounds, and $h^{s}$ and $h^{l}$ the acoustic response of the chest at the stethoscope location from the heart and lung sounds, respectively. However, no study has investigated the conjectured contribution of breathing sounds to heart auscultation and so far no standard filtering scheme has been proposed (Dalmay, Antonini, Marquet, \& Menier, 1995).

\section{THE TWO-STAGE CLASSIFICATION SYSTEM}

The heart sound signal and the vital signs provide partial indicators of the patient's cardiovascular health. As depicted in Figure 1, the proposed system is a two-stage classifier that analyzes the heart sound signals (using $\mathrm{CNN}$ ) and the vital signs (using decision trees) for a comprehensive initial assessment. The scoring scheme (Table 1) labels an individual "normal" if the heart sound signal and vital signs are both classified as "normal."

\subsection{Deep Learning for Heart Sound Anomaly Detection}

The problem of classifying heart sounds by deep learning is formulated as minimizing the loss function, defined as the difference between the target vector (i.e., ground truth labels) and predicted results (i.e., classifier outputs) (5). Let us assume that there are $N$ recordings comprising $c$ classes (in this research $c=2$, "normal" and "abnormal") and the recording length is represented by $D$ (in this research $D$ ranges from 4000 to 12,000 , translating to $2-6$ seconds at $f_{s}=2 \mathrm{kHz}$ ).

$$
\mathrm{x}=\left(x_{1}, \ldots, x_{D}\right) .
$$

The frequency domain signal with $N_{F F T}=256$ frequency bins calculated and averaged over 64 sample windows with a 32 -sample overlap is represented by:

Table 1: The proposed scoring scheme.

\begin{tabular}{l|lc} 
Heart sound & Vital signs & Score \\
\hline \hline Normal & Normal & Normal \\
Abnormal & Normal & $\begin{array}{c}\text { Possible anomaly } \\
\text { if recurring }\end{array}$ \\
Normal & Abnormal & $\begin{array}{c}\text { Possible anomaly } \\
\text { if recurring }\end{array}$ \\
Abnormal & Abnormal & Needs to be \\
& & examined by a \\
& & doctor
\end{tabular}

$$
x_{k}=\sum_{n=0}^{N-1} x(n) e^{\frac{-2 j \pi}{N_{F F T}} k n},
$$

where $k \in\left\{1, \ldots, N_{F F T}\right\}$.

$$
\mathrm{x}_{\mathrm{f}}=\left(x_{f 1}, \ldots, x_{f N_{F F T}}\right) \text {, }
$$

It is observed that normal heart sounds have distinct peaks (i.e., sharp edges correlated with higher frequencies) at $S 1$ and $S 2$, whereas abnormal heart sounds have weaker $S 1$ and $S 2$ edges distorted by strong murmurs (Figure 2). In the PhysioNet database, most abnormal instances are related to heart valve defects and coronary artery disease patients (PhysioNet, 2016). The presence of murmurs increases the heart sound complexity (Moukadem, Dieterlen, \& Brandt, 2013). This difference leads to a $30 \mathrm{~dB}$ difference (Figure 2) between the "normal" and the " abnormal" signal spectra. The binary classifier is trained by the time-domain (2) and frequency-domain (4) representations of the heart sound signals and by the ground-truth labels $y \in$ \{y_Normal,y_Abnormal\} provided by the applied database (PhysioNet, 2016). For a database consisting of $\mathrm{N}$ persons' data, the matrix of the heart sound recordings is $\mathrm{X}_{-} \mathrm{hs} \in \mathrm{R}^{\wedge}(\mathrm{N} \times \mathrm{D})$ and the target vector is $\mathrm{y} \_\mathrm{hs} \in \mathrm{B} \wedge(\mathrm{N} \times 1)$.

$$
\mathbf{X}_{h s}=\left(\begin{array}{ccc}
x_{1,1} & \ldots & x_{1, D} \\
\vdots & \ddots & \vdots \\
x_{N, 1} & \ldots & x_{N, D}
\end{array}\right)
$$

The predictor's matrix $\mathbf{X}_{h s}$ as in (4) and the target $\mathbf{y}_{h s}$ are applied to train the network, which is mathematically modeled as:

$$
\begin{aligned}
& \min \left(\ell\left(Y, \emptyset\left(X, W^{1}, \ldots, W^{k}\right)\right)\right. \\
& \left.+\lambda \theta\left(W^{1}, \ldots, W^{k}\right),\left\{W^{k}\right\}_{k=1}^{K}\right)
\end{aligned}
$$

where $\ell(Y, \emptyset)$ is a loss function measuring the agreement between the true output $\boldsymbol{y}$ and the predicted output.

$\emptyset\left(\mathbf{X}_{h s}, \mathbf{W}\right)$. The predicted label for each sample recording $x$ from (1) (rows of $\mathbf{X}$ ) is:

$$
y=\Psi \mathbf{x W}
$$

where $\Psi$ and $\mathbf{W}$ represent the bias and weights of the deep network, respectively. Table 2 summarizes the applied deep network architecture of the CNN that accepts a matrix (i.e., the transformed time-domain or frequency-domain vector) as input. A standard sound classification architecture is applied consisting of three convolutional layers, each followed by batchnormalization and max-pooling layers, followed by two fully connected layers before the final classification (Kim, Lee, \& Nam, 2018). The applied dataset $\left(\mathbf{X}_{h s}\right)$ includes 3126 normal and abnormal 
heart sound recordings sampled at $f_{s}=2000 \mathrm{~Hz}$. The length of the database recordings (PhysioNet, 2016) varies, but to maintain consistency, all the experiments are done with training sets and test sets of the same lengths (i.e., $2 s, \ldots, 6 s)$. Having $x(n)$

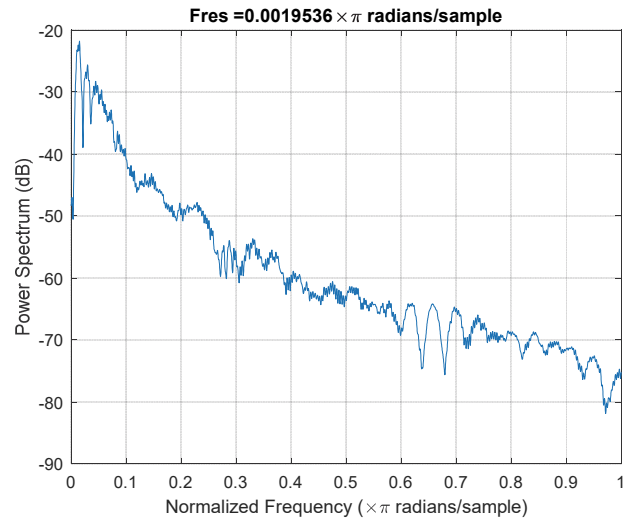

a)

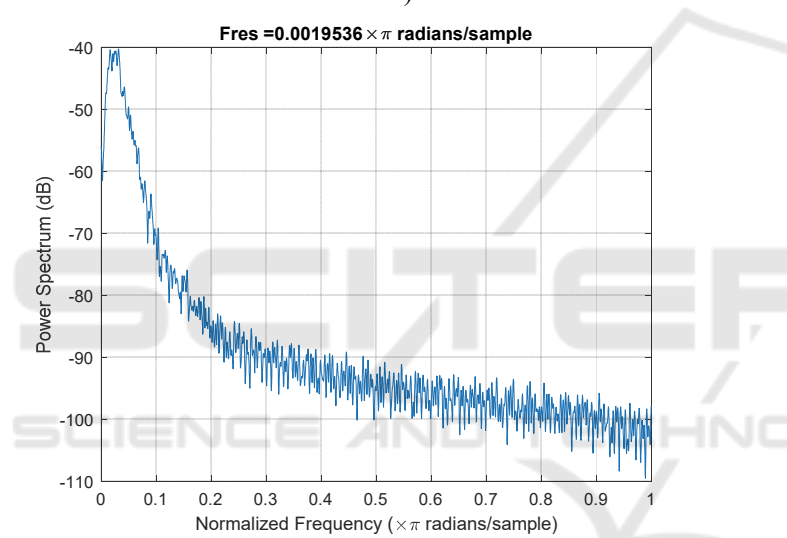

b)

Figure 2: Power spectrum of a) normal heart sound with distinct peaks and $b$ ) weaker abnormal heart sound.

Table 2: Summary of the CNN configuration.

\begin{tabular}{llccc} 
Layer & \multicolumn{1}{c}{ Type } & Output & $\begin{array}{c}\text { Kernel } \\
\text { size }\end{array}$ & Stride \\
\hline 1 & Convolution & $128 \times 8 \times 8$ & 8 & 1 \\
\hline 2 & BatchNorm & $128 \times 8 \times 8$ & - & - \\
\hline 3 & $\begin{array}{l}\text { Max } \\
\text { pooling }\end{array}$ & $64 \times 4 \times 8$ & 4 & 2 \\
\hline 4 & Convolution & $64 \times 4 \times 8$ & 8 & 1 \\
\hline 5 & BatchNorm & $64 \times 4 \times 8$ & - & - \\
\hline 6 & $\begin{array}{l}\text { Max } \\
\text { pooling }\end{array}$ & $32 \times 2 \times 8$ & 4 & 2 \\
\hline 7 & Convolution & $32 \times 2 \times 8$ & 8 & 1 \\
\hline 8 & BatchNorm & $32 \times 2 \times 8$ & - & - \\
\hline 9 & $\begin{array}{l}\text { Max } \\
\text { pooling }\end{array}$ & $12 \times 1 \times 8$ & 4 & 2 \\
\hline 10 & Flatten & 96 & - & - \\
\hline 11 & Softmax & 2 & - & - \\
\hline
\end{tabular}

from (1), the low-pass $\left(g_{L}\right)$ and high-pass $\left(g_{H}\right)$ filtered signals are given by:

$$
\hat{x}(n)=g_{L}(n) * x(n) .
$$

The training set $\mathbf{A}_{h s} \in \mathbb{R}^{M \times D}, M \cong 0.7 \times N$ consists of $70 \%$ of the dataset. The heart sound vectors are transformed into matrices to match the 2D input layer of the CNN (Table 2) The initial learning rate is set to 0.01 with a maximum of 250 epochs. All convolution layers are zero padded to preserve the input dimension.

\subsection{Decision Tree for the Vital Signs}

Let $\left\{\mathbf{X}_{v i}, \mathbf{y}_{v i}\right\}$ denote the vital signs of $N$ individuals and the ground-truth targets; $\boldsymbol{\theta}$ represents the classification parameters and $C$ the cost function. The goal of the classification problem is to find the classifier $f\left(\mathbf{x}_{v i}, \widehat{\boldsymbol{\theta}}\right)$

where $\widehat{\boldsymbol{\theta}}=\arg \min _{\theta, \mathrm{X}} \mathrm{C}(\boldsymbol{\theta})$. Thirteen vital signs (Z)

(University of California Irvine Machine Learning Repository, n.d.) associated with heart defects are considered in this research. Medical experts believe that these 13 vital signs and additional patient information can help in understanding the heart defect cause and type, if measured or provided as side information during a cardiovascular examination.

The $\mathrm{C} 4.5$ decision tree (i.e., the advanced version of the ID3 algorithm) is applied in this work to create models including both continuous numerical data (e.g., age and serum cholesterol) and categorical data (e.g., sex and chest pain type). The process of inducing the decision tree includes calculating the information gain and the gain ratio of each feature, which shows the significance of each feature for the classification. Finding the right threshold for each feature depending on the data distribution is also part of the inducing process. The applied dataset includes thirteen features ( Z ) of 270 adults (i.e., 300 augmented instances). There are $\left(\begin{array}{c}13 \\ 1\end{array}\right)+\cdots+$ $\left(\begin{array}{l}13 \\ 13\end{array}\right)=1891$ possible combinations of features used in performing the classification. The feature selection process of this research (Table 4) concludes that using only the six features highlighted in Table 3 outperforms all other possible combinations and also reduces the computation cost. This feature selection improves the classification accuracy (11) by 11 percentage points from $72 \%$ to $83 \%$ compared with the scenario in which all thirteen features are applied. Information gain and entropy are applied to decide the most significant vital signs. The entropy of each vital sign is defined as: 


$$
H\left(\mathbf{X}_{v i}\right)=-\sum_{c} p_{c} \log _{2} p_{c}
$$

where $p_{c}=\frac{N_{c}}{N}$ is the probability of each class in $\mathbf{X}_{v i}$. Using $H$, the information gain $(\Delta H)$ for a vital sign $(z \in \mathrm{Z})$ is given by:

$$
\Delta H=H\left(\mathbf{X}_{v i}\right)-H\left(\mathbf{X}_{v i}, z\right)
$$

where $H\left(\mathbf{X}_{v i}, z\right)$ is the entropy of $\mathbf{X}_{v i}$ classified according to the feature $z$. Accuracy and other classification measurements are then calculated by:

$$
\begin{gathered}
\text { Accuracy }=\frac{T_{P}+T_{N}}{T_{P}+T_{N}+F_{N}+F_{P}}, \\
\text { Sensitivity }=T P R=\frac{T_{P}}{T_{P}+F_{N}}, \\
\text { Specificity }=T N R=\frac{T_{N}}{T_{N}+F_{P}}, \\
\text { Precision }=P P V=\frac{T_{p}}{T_{p}+F_{p}}, \\
\text { Fscore }=2 \times \frac{P P V \times T P R}{P P V+T P R} .
\end{gathered}
$$

Table 3: The applied vital signs and patient data.

\begin{tabular}{|l|c|}
\hline \multicolumn{1}{|c|}{ Vital sign } & $\begin{array}{c}\text { Information } \\
\text { gain }\end{array}$ \\
\hline 1. Age & 0.015 \\
2. Sex* & $\mathbf{0 . 2 2 2}$ \\
3. Chest pain* & $\mathbf{0 . 2 6 6}$ \\
4. Resting blood pressure & 0.015 \\
5. Serum cholesterol & 0.052 \\
6. Fasting blood sugar & 0.008 \\
7. Resting electrocardiographic results & 0.060 \\
8. Maximum heart rate achieved & 0.079 \\
9. Exercise-induced angina * & $\mathbf{0 . 1 2 0}$ \\
10. Old peak & 0.089 \\
11.Slope of the peak exercise ST & $\mathbf{0 . 1 9 4}$ \\
segment * & \\
12. Number of major vessels colored & $\mathbf{0 . 1 8 8}$ \\
by fluoroscopy* & $\mathbf{0 . 2 1 8}$ \\
13. Thalassemia * &
\end{tabular}

Table 4: Feature selection algorithm.

Calculate $\Delta H$ (9) for all the vital signs Z using $\mathbf{X}_{v i}$ and $\mathbf{y}_{v i}$

Sort the features based on their $\Delta H$ (Table 3 ) in descending order $(\hat{\mathrm{Z}})$.

For $i=2$ to 13 (length of $(\hat{\mathrm{Z}})$ ):

- Classify the test set using the first $i$ features from $(\widehat{\mathrm{Z}})$ and calculate the classification accuracy (Accuracy $(i)) \quad(10)$

- $\quad$ Stop the process if Accuracy $(i) \leq \operatorname{Accuracy}(i-$ 1)

\section{EVALUATION AND DISCUSSION}

The CNN classifier and the decision tree are evaluated using unseen samples that were excluded from the training set. The effect of the heart sound recording lengths, filtering and feature selection are investigated in the evaluation process. As the two stages consume different types of signals they are independently trained and evaluated.

\subsection{CNN Evaluation}

The trained CNN was able to correctly classify an average of $74.19 \% \quad$ (10) of 660 unseen samples of different frame lengths (Figure 3) from the database and from recordings made using a Thinklabs One digital stethoscope (Thinklabs, n.d.) down-sampled from $44.1 \mathrm{kHz}$ to $2 \mathrm{kHz}$. The F-score (14) is calculated for the time-domain (1) and frequencydomain signals (3) in Figure 3 for different test set frame lengths. It is important to note that the false negatives (i.e., individuals with heart issues who are not detected by the classifier) are the main concern of medical experts, and the goal of the assessment process is to minimize the false negative rate, i.e., $F N R=1-T P R \quad$ (11). It is shown that the minimum required heart sound auscultation length for obtaining reliable results is 5 6 seconds (Figure 4).

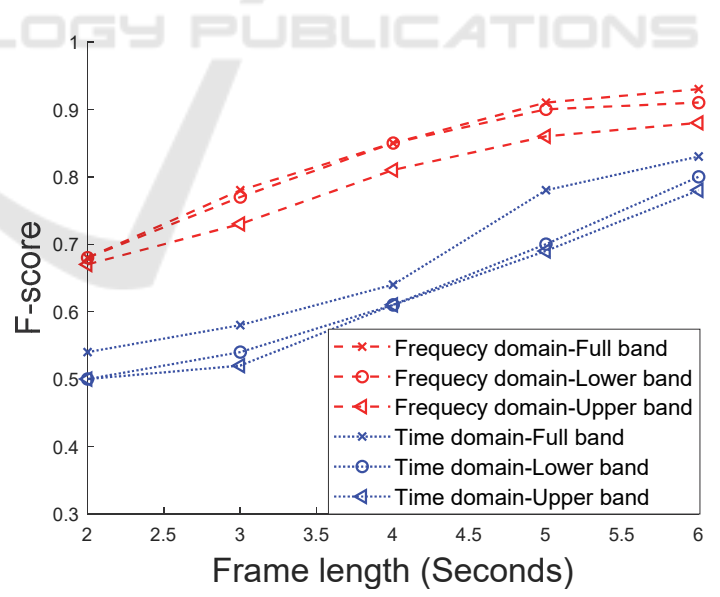

Figure 3: Heart sound signal classification evaluation by CNN.

A high-pass filter $(500-1000 \mathrm{~Hz})$ and a low-pass filter $(0-500 \mathrm{~Hz})$ with $80 \mathrm{~dB}$ attenuation per octave are applied to coarsely find the more discriminative frequency band. A more thorough investigation of the frequency bands is conducted by using low-pass and high-pass filters with varying pass-band frequencies 
ranging from a minimum of $200 \mathrm{~Hz}$ to a maximum of $800 \mathrm{~Hz}$ (Figure 5). The results suggest that using a high-pass filter with a pass-band frequency of $200 \mathrm{~Hz}$ (i.e., suppressing the $0-200 \mathrm{~Hz}$ band) yields the highest classification accuracy. This could be due to removing the low-frequency noise from the respiratory system while passing the discriminative components of the murmur and heartbeat signals.

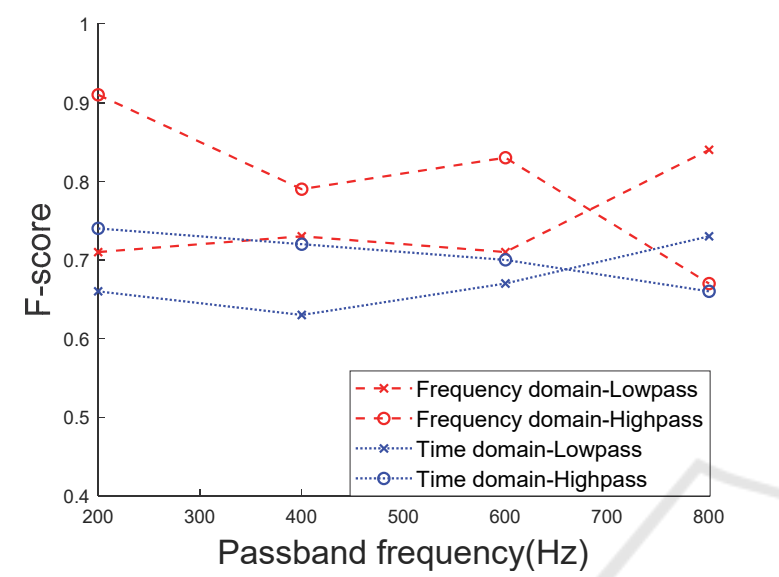

Figure 4: Classification results for filtered four-second signals.

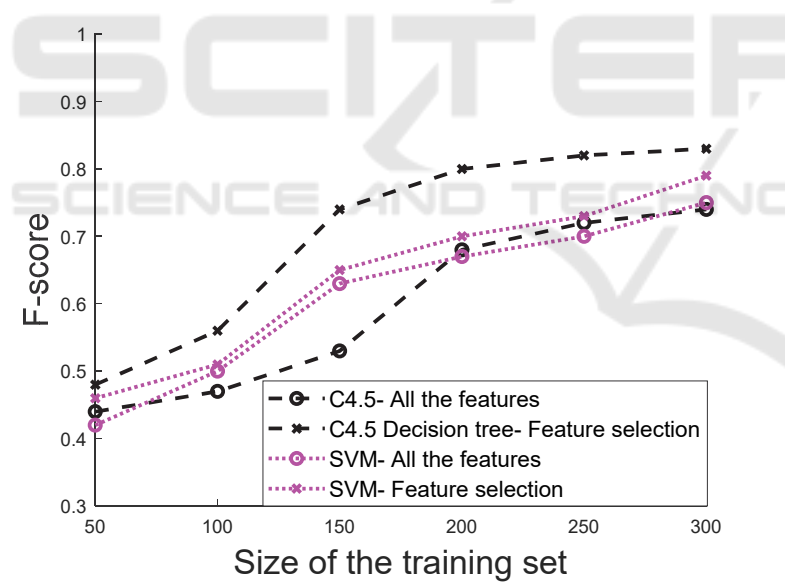

Figure 5: Vital sign classification and feature selection evaluation.

\subsection{Decision Tree Evaluation}

The induced C4.5 decision tree successfully classified $83 \%$ (feature selection enabled) and $72 \%$ (using all features) of the unseen vital signs from the test set. The F-score is calculated to evaluate the classification accuracy, including both precision and recall. The results are compared with those of the baseline support vector machine (SVM) usually used as a binary classifier. It is shown that the $\mathrm{C} 4.5$ classifier outperforms the baseline SVM for all training set sizes (Figure 6).The experimental studies also investigated the significance of feature selection (Visalakshi \& Radha, 2014) to find the optimal set yielding the highest classification accuracy, which could be of considerable interest for large healthcare project data collection. It is observed that using only the six selected features (highlighted in ) improves the F-score by an average of 0.2 for the $\mathrm{C} 4.5$ classifier.

$$
\begin{gathered}
\text { Precision }=P P V=\frac{T_{p}}{T_{p}+F_{p}}, \\
F \text { score }=2 \times \frac{P P V \times T P R}{P P V+T P R} .
\end{gathered}
$$

\section{CONCLUSION}

This research reported an implementation of an AI system trained for cardiovascular examination decision support. The aim of the project was to analyze the heart sound signals and patient information to produce a reliable, comprehensive, and time-efficient screening. The results suggest that the proposed two-stage approach provides accurate suggestions that correctly classify a maximum of $91 \%$ of the heart sound signals and $83 \%$ of the accompanying vital signs and information. Although using the time-domain and frequency-domain representations of the heart sound signals did not improve the results compared with the mathematical and statistical features applied in the PhysioNet challenge, using the side information and the vital signs within a two-stage approach increased the screening reliability. Future work will investigate more specialized deep network architectures for diagnosing each heart defect type.

\section{REFERENCES}

Amiriparian, S., Schmitt, M., Cummins, N., Qian, K., Dong, F., \& Schuller, B. (2018). Deep unsupervised representation learning for abnormal heart sound classification. 40th Annual International Conference of the IEEE Engineering in Medicine and Biology Society (EMBC). 18-21 July, Honolulu, USA.

Barhatte, A., Ghongade, R., \& Thakare, A. (2015). QRS complex detection and arrhythmia classification using SVM. 2015 Communication, Control and Intelligent Systems (CCIS). 7-8 Nov., Mathura, India.

Chen, Q., Zhang, W., Tian, X., Zhang, X., Chen, S., \& Lei, W. (2016). Automatic heart and lung sounds classification using convolutional neural networks. 2016 Asia-Pacific Signal and Information Processing 
Association Annual Summit and Conference. 13-16 Dec., Jeju, Korea.

Chen, T. (2017). S1 and S2 heart sound recognition using deep neural networks. Transactions on Biomedical Engineering, 64(2), 372-380.

Clifford, G. (2016). Classification of normal/abnormal heart sound recordings: The PhysioNet/Computing in Cardiology Challenge 2016. 2016 Computing in Cardiology Conference (CinC). 11-14 Sept., Vancouver, BC, Canada.

Dalmay, F., Antonini, M., Marquet, P., \& Menier, R. (1995). Acoustic properties of the normal chest. European Respiratory Journal, 8(10), 1761-1769.

Gharehbaghi, A., \& Lindén, M. (2017). A deep machine learning method for classifying cyclic time series of biological signals using time-growing neural network. Transactions on Neural Networks and Learning Systems, 29(9), 4102-4115.

IBM Watson. (n.d.). Retrieved 23 September 2019 from https://www.ibm.com/watson

Islam Chowdhuryy, M., Sultana, M., Ghosh, R., Ahamed, J., \& Mahmood, M. (2018). AI assisted portable ECG for fast and patient specific diagnosis. International Conference on Computer, Communication, Chemical, Material and Electronic Engineering (IC4ME2). 8-9 Feb., Rajshahi, Bangladesh.

Ivascu, T., Cincar, K., \& Negru, V. (2017). Activities of daily living and falls recognition and classification from the wearable sensors data. 2017 E-Health and Bioengineering Conference (EHB). 22-24 June, Sinaia, Romania.

Jones, B. (2013). Developing a vital sign alert system. The American Journal of Nursing, 8(113), 36-44.

Kim, T., Lee, J., \& Nam, J. (2018). Sample-level CNN architectures for music auto-tagging using raw waveforms. ICASSP 2018 - 2018 IEEE International Conference on Acoustics, Speech and Signal Processing (ICASSP). 15-20 April, Calgary, AB, Canada.

Lin, C., Chang, Y., Lin, C., Tsai, L., \& Chen, J. (2017). Development of an AI-based non-invasive Pulse AudioGram monitoring device for arrhythmia screening. 2017 IEEE Healthcare Innovations and Point of Care Technologies (HI-POCT). 6-8 Nov., Bethesda, MD, USA.

Loh, B., \& Then, P. (2017). Deep learning for cardiac computer-aided diagnosis: benefits, issues \& solutions. Mhealth, 3(45), doi: 10.21037/mhealth.2017.09.01.

Morsy, A. (2018). Can AI truly transform health care? A recent IEEE Pulse on Stage forum offers some perspective. IEEE Pulse, 9(4), 18-20.

Moukadem, A., Dieterlen, A., \& Brandt, C. (2013). Shannon Entropy based on the S-Transform Spectrogram applied on the classification of heart sounds. 2013 IEEE International Conference on Acoustics, Speech and Signal Processing. 26-31 May, Vancouver, BC, Canada.

Official Website of the Office of the National Coordinator for Health Information Technology (ONC). (n.d.).
Retrieved $23 \quad$ September 2019 from https://www.healthit.gov/topic/health-itinitiatives/blue-button

PhysioNet. (2016). Retrieved 26 February 2019 from https://physionet.org/challenge/2016/

Shao, L., Wu, D., \& Li, X. (2014). Learning deep and wide: A spectral method for learning deep networks. IEEE Transactions on Neural Networks and Learning Systems, 25(12), 2303-2308.

Thinklabs. (n.d.). Retrieved 3 October 2019 from https://www.thinklabs.com/one-digital-stethoscope

Thomae, C., \& Dominik, A. (2016). Using deep gated RNN with a convolutional front end for end-to-end classification of heart sound. 2016 Computing in Cardiology Conference (CinC). 11-14 Sept., Vancouver, BC, Canada.

University of California Irvine Machine Learning Repository. (n.d.). (Center for Machine Learning and Intelligent Systems) Retrieved 1 October 2019 from http://archive.ics.uci.edu/ml/datasets/statlog+(heart)

Vepa, J. (2009). Classification of heart murmurs using cepstral features and support vector machines. 2009 Annual International Conference of the IEEE Engineering in Medicine and Biology Society. 3-6 Sept., Minneapolis, MN, USA.

Vernekar, S., Nair, S., Vijaysenan, D., \& Ranjan, R. (2016). A novel approach for classification of normal/abnormal phonocardiogram recordings using temporal signal analysis and machine learning. 2016 Computing in Cardiology Conference (CinC). 11-14 Sept., Vancouver, BC, Canada.

Visalakshi, S., \& Radha, V. (2014). A literature review of feature selection techniques and applications: Review of feature selection in data mining. 2014 IEEE International Conference on Computational Intelligence and Computing Research. 18-20 Dec., Coimbatore, India.

Yamashita, M., Himeshima, M., \& Matsunaga, S. (2014). Robust classification between normal and abnormal lung sounds using adventitious-sound and heart-sound models. 2014 IEEE International Conference on Acoustics, Speech and Signal Processing (ICASSP). 4 9 May, Florence, Italy.

Zabihi, M., Rad, A., Kiranyaz, S., Gabbouj, M., \& Katsaggelos, A. (2016). Heart sound anomaly and quality detection using ensemble of neural networks without segmentation. 2016 Computing in Cardiology Conference (CinC). 11-14 Sept., Vancouver, BC, Canada. 